\title{
Effective measures to foster girls' interest in secondary computer science education
}

\section{A Literature Review}

\section{Lucia Happe $^{1}$ (D) $\cdot$ Barbora Buhnova ${ }^{2} \cdot$ Anne Koziolek $^{1} \cdot$ Ingo Wagner $^{1}$}

Received: 3 June 2020 / Accepted: 27 October 2020 / Published online: 16 November 2020

(C) The Author(s) 2020

\begin{abstract}
The interest of girls in computing drops early during primary and secondary education, with minimal recovery in later education stages. In combination with the growing shortage of qualified computer science personnel, this is becoming a major issue, and also a target of numerous studies that examine measures, interventions, and strategies to boost girls' commitment to computing. Yet, the results of existing studies are difficult to navigate, and hence are being very rarely employed in classrooms. In this paper, we summarize the existing body of knowledge on the effective interventions to recruit and retain girls in computer science education, intending to equip educators with a comprehensive and easy-to-navigate map of interventions recommended in the existing literature. To this end, we perform an aggregated umbrella literature review of 11 existing reviews on the topic, together accumulating joined knowledge from over 800 publications, and formulate the findings in a map of 22 concrete interventions structured in six groups according to their phase and purpose.
\end{abstract}

Keywords Computer science education · Girls · Interventions · Motivation · Interest $\cdot$ Self-efficacy

\section{Introduction}

As the world transitions to an increasingly digital economy, most countries lack qualified personnel in jobs related to computer science, a shortage that sheds light on

Lucia Happe

lucia.happe@kit.edu

Barbora Buhnova

buhnova@fi.muni.cz

1 Karlsruhe Institute of Technology, Karlsruhe, Germany

1 Masaryk University, Brno, Czech Republic 
critically low representation of women in this industry (Powell and Chang 2016). Despite interventions over the past decade, the gender gap in computing seems to be increasing and worldwide, almost everywhere fewer than one in five computer science graduates are female (Schlegel 2016). This urges fundamental changes in the way we approach early education of girls in computer science and STEM (Science, Technology, Engineering and Mathematics) (Gorbacheva et al. 2019), as the declining interest of girls in these disciplines seems to be preventable through tailoring education to girls' specific needs to help them embrace computing (Accenture 2016).

While numerous studies examine the narrative and root causes of the low engagement of girls in computer science education (Craig 2016; Barker and Aspray 2006; Lye and Koh 2014; García-Peñalvo et al. 2016; Siiman et al. 2014), comprehensive guidance for assisting educators in navigating the findings and recommendations published in literature is missing (Craig 2016; Barker and Aspray 2006; Murphy et al. 2019). Identification of practical and suitable guidelines is challenging, as the individual studies vary in scope, education stage (primary, secondary, tertiary), goals, and also results (Gorbacheva et al. 2019; Craig 2016; Main and Schimpf 2017). Moreover, because of the unclear goal of the known interventions, strategies and measures in the classroom, resulting in some cases from the vague definition of targeted changes (e.g., increased motivation or change of perception), it is especially hard to decide what are the necessary interventions to retain the attention of girls during the school or non-school computer science lessons.

This paper aims to provide orientation in the recommendations for computer science educators committed to sparking and retaining the interest and participation of girls in their classes. This guidance is extracted from existing reviews on the topic in terms of an aggregated study (review of reviews). As the secondary education is the life-altering time period, where the presumed self-selection of girls away from computer science and other STEM subjects happens (Murphy et al. 2019), we have chosen this period as the key period of the study. Altogether, we have formulated four research questions, and on the way to responding to them, we have extracted findings from 11 existing comprehensive reviews on the topic, each giving a partial view of the problem, but together allowing us to form a compact overview of 22 measures and strategies towards our goal.

The rest of the paper is structured as follows. After discussing the methodology of our aggregated review in Section 2, we present the identified effective measures and strategies in Section 3. Section 4 discusses reflections from the performed study together with identified gaps in existing research, and Section 5 concludes the paper.

\section{Methodology}

Since numerous comprehensive studies have attempted to map the state of the art of the measures of girls engagement within computer science education (Brotman and Moore 2008; Siiman et al. 2014; Boston and Cimpian 2018), we have decided to adopt a systematic aggregated review method by Gough et al. (2013) and Aromataris et al. (2015), who suggest approaching a systematic literature review via combining 
existing reviews with the later synthesis of further publications in the time period following the date of publication of the identified reviews.

Examining existing reviews is, in general, an efficient and pragmatic way to mapping existing works and synthesizing their findings. It is also associated with challenges and limitations. Although aligned in their topic, the reviews might target different research questions, examine different target groups, or differ in their timeline coverage. Such an aggregated, umbrella review might hence not be fully complete. Despite that, thanks to the broad coverage of an extensive number of works and comprehensive discussion of findings in each of the reviews, an aggregated, umbrella review is a very effective review strategy, which is also reliable in its findings if well executed.

In the initial step of the literature review, we identified recent literature reviews and filtered them according to the predefined inclusion and exclusion criteria. We focused on the reviews with the highest impact, best coverage, and highest clarity in their methodology. Later, we aggregated the results presented in the reviews and formed a set of recommendations for educators and researchers. We structured and organized the recommendations in a compact overview based on their purpose and phase of impact on the educational outcome.

\subsection{Research questions}

Before setting the criteria for the literature search, we have formulated the following research questions:

- RQ1: What measures foster girls' interest in computer science (CS) education?

- RQ2: Are the recommended measures across the reviewed literature consistent (non-contradictory)?

- RQ3: What are the minimum recommended practices to create girl-friendly CS classroom, including recommendations for curriculum as well as the environment?

- RQ4: Are there gaps in the examined measures and strategies that would deserve better research coverage?

\subsection{Literature search}

The reviews covered within our aggregated review have been gathered using search in the following research databases: ACM Digital Library, IEEE, Google Scholar, Springer, Wiley Online Library, and Eric. Searches were conducted with the following search term, whose results were later filtered with our inclusion and exclusion criteria:

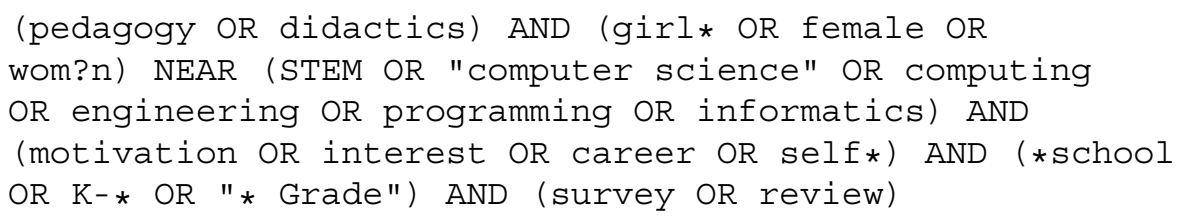




\subsection{Inclusion and exclusion criteria}

The initial search generated hundreds of articles on diverse topics targeted to understand the causes, the consequences, and the solutions to gender distribution in CS education and related parts of STEM education. We filtered the results and included the ones that are:

1. providing some insights or guidance on possible solutions to the related problems,

2. reporting research on CS education or career perception specifically in computing, computer science, software engineering, computer engineering, or informatics; and

3. focusing on girls or women in CS education, specifically including secondary education.

We include survey papers published after the year 2000, without limitation of their scope of review. We do not include the survey papers from the 90's, as these papers tend to focus on the problem of access to a computer, which is not relevant today (Barker and Aspray 2006). We also exclude studies that do not examine relevant interventions, such as the study by Barker and Aspray (2006), who reviewed social science literature from 1994 to 2004, studying the causes for the gender distribution, without interventions relevant to our aim.

\subsection{Included publications breakdown}

This filtering process yielded 11 comprehensive articles, thereof seven peer-reviewed literature reviews, and four other review-style studies, including a project deliverable, research report, book chapter, and Ph.D. thesis, which were included in our aggregated umbrella review. Table 1 summarises the reviews included in our study. The reviewed publications are together accumulating joined knowledge from over 800 publications.

Table 1 shows that the included reviews have different foci and, as such, provide a view on the problem from diverse standpoints, trying to achieve diverse goals. Therefore, although we investigate our research questions, when we describe the results from the included reviews, we use the original wording to report their concepts truthfully and unbiased. In our further analysis, when extracting any usable and practical knowledge of effective interventions, measures, and strategies to recruit and retain girls in CS education, we have observed that none of these reviews covers the problem fully (see Table 2) and hence the aggregated review is of high added value in this case.

\subsection{Results analysis}

In our review, we have followed the four research questions defined above. In response to RQ1, we used an aggregative approach to collect effective measures and strategies. In the reviews included in our study, insufficient or insufficiently similar 


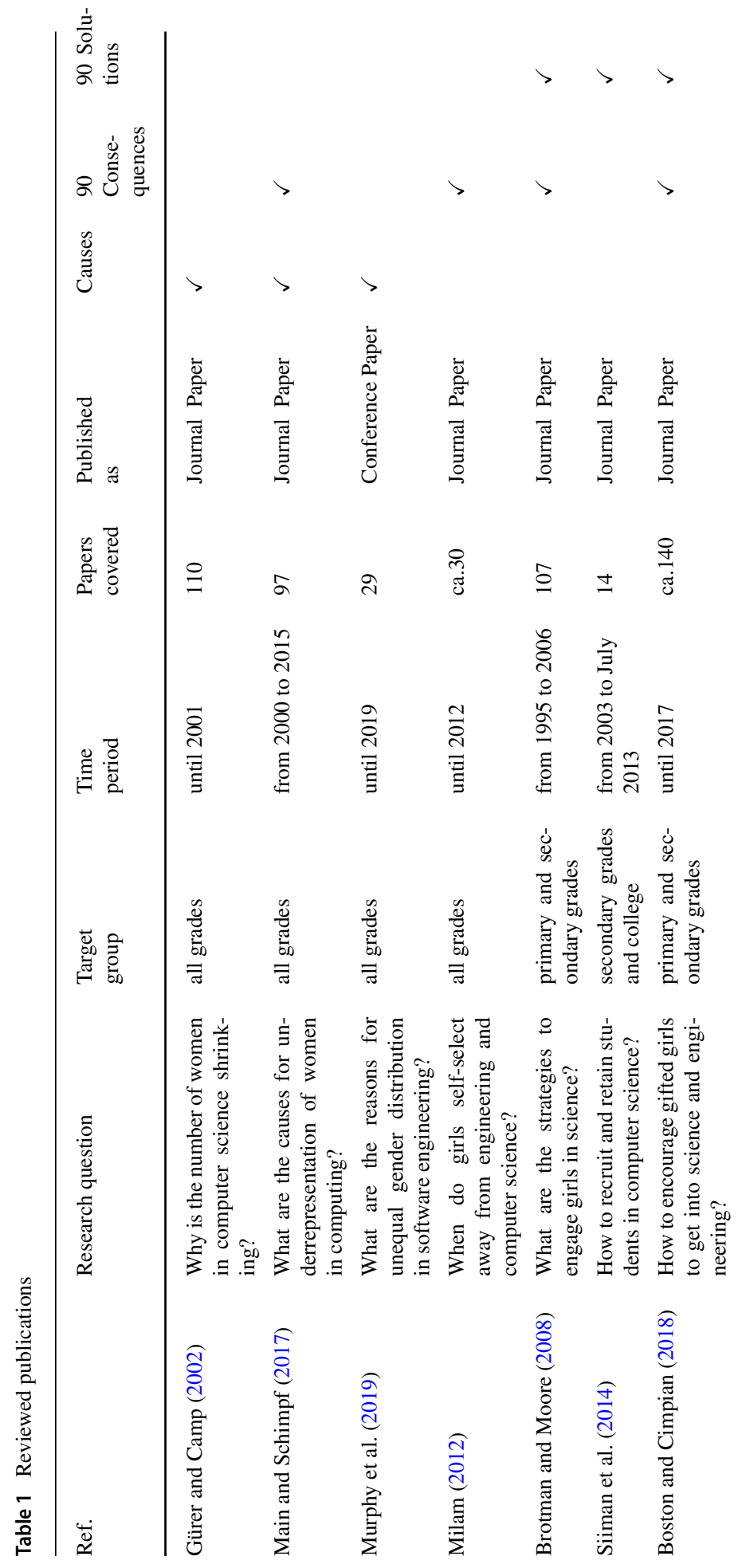




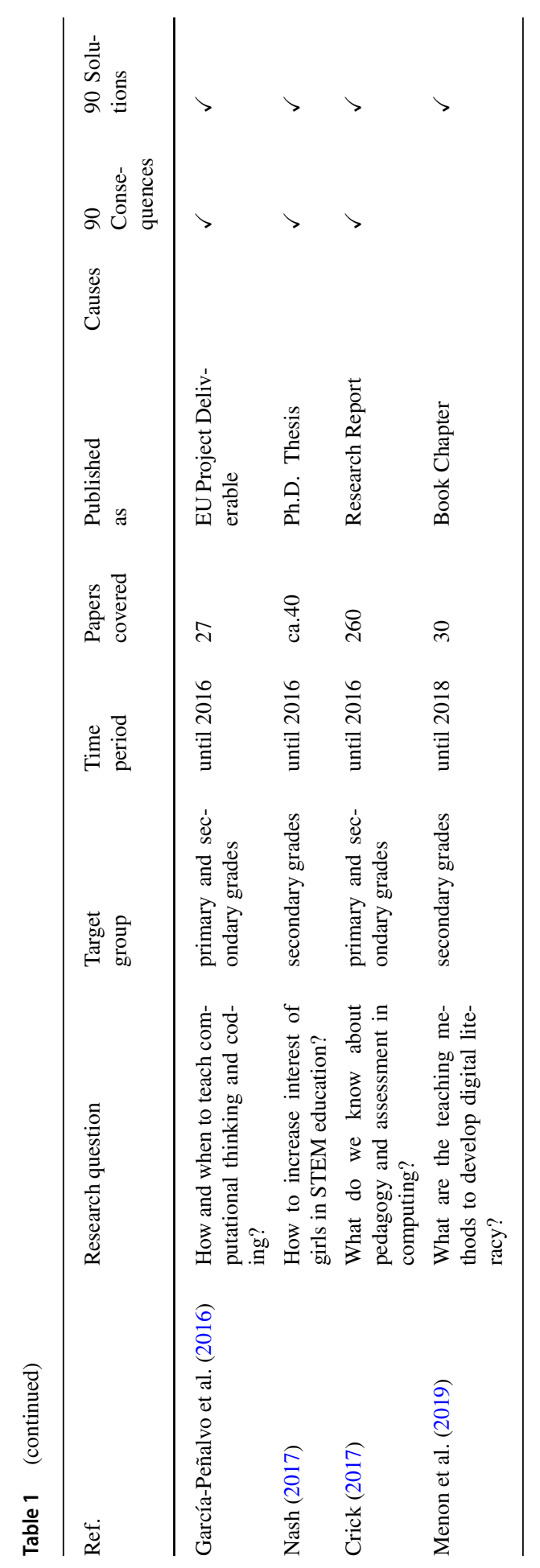


Table 2 Effective measures and strategies

(

$$
\text { Recommendation (Rec.) }
$$

Goal of the strategy

1. STRATEGIES THAT COMBAT WRONG STEREOTYPES

1.1. Provide non-stereotypical role models (Boston and Cimpian 2018)

1.2. Portray work in computing as helpful, altruistic, and community-oriented (Boston and Cimpian 2018)

1.3. Provide opportunities to do computing activities as part of a group (Boston and Cimpian 2018; Brotman and Moore 2008; Nash 2017)

1.4. Expose girls to successful and relatable female role models in computing (Boston and Cimpian 2018; Nash 2017)

\section{STRATEGIES THAT MOTIVATE AND} SPARK INITIAL INTEREST

2.1. Emphasising the social impact and interdisciplinary nature of computing work (Main and Schimpf 2017; Siiman et al. 2014; García-Peñalvo et al. 2016; Brotman and Moore 2008; Murphy et al. 2019; Crick 2017)

2.2. Include physical reminders of women's success in computing (Boston and Cimpian 2018)

3. STRATEGIES THAT PROVIDE SUITABLE FIRST CONTACT

3.1. Digital gaming and creative arts activities designed for girls (Main and Schimpf 2017; Milam 2012)

3.2. Start with offline contextualised activities (García-Peñalvo et al. 2016; Menon et al. 2019)

3.3. Visual programming environments to teach introductory programming (Siiman et al. 2014; Menon et al. 2019)

3.4. Involve discussion and reflections on the activities (García-Peñalvo et al. 2016)

4. STRATEGIES THAT MAKE LEARNING ENVIRONMENT LESS HOSTILE

4.1. All-female education programs and classes (Gürer and Camp 2002; García-Peñalvo et al. 2016)

4.2. Splitting classes by experience not gender (Siiman et al. 2014)

4.3. Non-competitive environment (Boston and Cimpian 2018)
Revising stereotypes

Revising what computer scientist do

Revising how computer scientist work

Inoculate girls against bad stereotypes ("psychological vaccines")

Increase sense of belonging, perceived similarity, relevance and opportunity to succeed

Providing role models

Provide entry point to computing for girls

Decrease barrier to start and practice

Providing engaging less rigorous introduction

Promote learning

Allowing girls to get more instructor attention

Working against monopolisation of instructor time by the most experienced students

Minimising building of classroom hierarchy hostile to girls 
Table 2 (continued)

\begin{tabular}{|c|c|c|}
\hline & Recommendation (Rec.) & Goal of the strategy \\
\hline 4.4. & $\begin{array}{l}\text { Collaborative assignments (Boston and } \\
\text { Cimpian 2018; Brotman and Moore 2008; } \\
\text { Nash 2017) }\end{array}$ & $\begin{array}{l}\text { Broadening spectrum of skills and experi- } \\
\text { ences gained in classroom }\end{array}$ \\
\hline 5. & $\begin{array}{l}\text { STRATEGIES THAT BUILD SELF- } \\
\text { CONFIDENCE }\end{array}$ & \\
\hline 5.1 & $\begin{array}{l}\text { Providing low-stakes opportunities for girls } \\
\text { to experience success (Boston and Cimpian } \\
\text { 2018; Nash 2017) }\end{array}$ & Gaining confidence \\
\hline 5.2. & $\begin{array}{l}\text { Install a growth mind-set the belief that abil- } \\
\text { ities can be improved with effort, strategies, } \\
\text { and mentoring (Boston and Cimpian 2018) }\end{array}$ & $\begin{array}{l}\text { Inoculate girls against bad stereotypes and } \\
\text { hostile culture ("psychological vaccines") }\end{array}$ \\
\hline 5.3 . & $\begin{array}{l}\text { Adopt a positive, constructive attitude } \\
\text { toward failure as a valuable learning oppor- } \\
\text { tunity (Boston and Cimpian 2018) }\end{array}$ & $\begin{array}{l}\text { Inoculate girls against hostile culture ("psy- } \\
\text { chological vaccines") }\end{array}$ \\
\hline 6. & $\begin{array}{l}\text { STRATEGIES FOR SUSTAINING LONG- } \\
\text { TERM INTEREST }\end{array}$ & \\
\hline 6.1. & $\begin{array}{l}\text { Education of teachers providing them with } \\
\text { tools and approaches to better engage girls } \\
\text { (Brotman and Moore 2008; Nash 2017; } \\
\text { Crick 2017) }\end{array}$ & $\begin{array}{l}\text { Increase ability of teachers to prevent girls } \\
\text { disengagement }\end{array}$ \\
\hline 6.2. & $\begin{array}{l}\text { Interesting and engaging real-world inquiry- } \\
\text { based open-ended hand-on experiences } \\
\text { (Main and Schimpf 2017; Siiman et al. } \\
\text { 2014; Brotman and Moore 2008; Nash } \\
\text { 2017) }\end{array}$ & $\begin{array}{l}\text { Having impact, inquiry and achievement } \\
\text { motivate girls more as challenge }\end{array}$ \\
\hline 6.3 . & $\begin{array}{l}\text { Emphasising the social impact and interdis- } \\
\text { ciplinary nature of computing work (Main } \\
\text { and Schimpf 2017; Siiman et al. 2014; } \\
\text { García-Peñalvo et al. 2016; Brotman and } \\
\text { Moore 2008; Murphy et al. 2019; Crick } \\
\text { 2017) }\end{array}$ & $\begin{array}{l}\text { Increase sense of belonging, perceived simi- } \\
\text { larity, relevance and opportunity to succeed }\end{array}$ \\
\hline 6.4. & $\begin{array}{l}\text { Provide long-term self-directed projects } \\
\text { (Brotman and Moore 2008) }\end{array}$ & Gaining experience with self-efficacy \\
\hline 6.5 . & $\begin{array}{l}\text { Community and being part of group (Boston } \\
\text { and Cimpian 2018; Brotman and Moore } \\
\text { 2008; Nash 2017) }\end{array}$ & Increase the sense of belonging \\
\hline
\end{tabular}

data were available to allow a statistical meta-analysis. This hindered the statistical synthesis of results from the reviews.

As the reviews vary in sample size and quality of execution, it could be misleading to simplify it to 'vote count' on effective strategies. Nevertheless, looking at patterns and common recommendations in the reviews showed to be very fruitful. We aggregated our findings into 22 concrete interventions structured in seven groups according to their phase and purpose, and present these in Table 2. Based on this phase, we also answer RQ2 and RQ3, which are both examined in Section 3 together with RQ1. 
RQ4 is addressed in Section 4, which suggests the directions for future research on the topic.

\section{Effective measures and strategies}

While existing literature primarily examines the likely causes and consequences of the underrepresentation of women in computing, efforts are emerging that address and alleviate these gender inequities at all levels. We have focused our research on identifying and summarising the effective measures and strategies to foster girls' interest in CS education (RQ1), targeted on increasing at least one of motivation, interest, career perception, self-confidence or self-efficacy, as well as retention of girls in CS.

After extracting effective measures and strategies recommended by the reviewed studies in the context of secondary education, we have clustered them according to their purpose, and present them in a comprehensive overview in Table 2, which links each recommended measure or strategy to the reviews that suggest it.

Our results show that one of the most powerful elements, resulting in student participation and retention, is interest (Krapp and Prenzel 2011; Ainley 2007; Bull et al. 2010). Interest energizes the learning process, guides the learning trajectory, and is crucial for the success of the overall endeavor. We hence organized the identified recommendations according to the phases in which interest is cultivated and evolves into confidence and commitment. Table 2 maps this conceptual model of interest emergence showing how interventions promote its development and subsequent goals in six chronological phases:

- Table 2 - Rec. 1: The first group of measures targets the time before the first contact with computing and combats wrong stereotypes when the psychological state could be impacted, and students could disengage with the topic before experiencing it.

- Table 2 - Rec. 2: The second group of measures triggers initial situational interest. The goal of the measures is to stimulate attention and engagement.

- Table 2 - Rec. 3: The third group includes strategies that provide suitable first contact. These strategies aim to maintain situational interest and allow easy to slide into practicing and experiencing the topic.

- Table 2-Rec. 4: The fourth group consists of strategies that make learning environment less hostile. These strategies target, minimize, or inoculate girls against the experiences in the classroom or outside of it that could lead to disengagement.

- Table 2 - Rec. 5: The fifth group consists of strategies that increase the occurrence of repeated experiences that maintain situational interest, which can develop into an individual interest, motivating the individual to seek opportunities to reengage with the topic, while building confidence about it.

- Table 2 - Rec. 6: The sixth group of strategies builds sustainable long-term interest and enduring predisposition to reengage over time. These strategies result in a well-developed individual interest and commitment. 
Although the recommendations are scattered across all the reviewed sources (i.e., no source would give a comprehensive overview of all, not even covering all the individual phases/recommendation groups), no contradictions among the recommendations were identified. In this sense, we consider the examined reviews consistent (RQ2), although each review appears to be incomplete in some sense. That is a natural consequence of variations in their target group (what period of life they examine) and goals (research questions they address).

As for the target group, variations can be identified between lower secondary education (i.e., middle school, pre-high school, typically age 11-15) and upper secondary education (i.e., high-school, pre-college, typically age 16-19). Many of the interventions designed for lower-secondary populations aim to increase girls' initial interest in computing (Main and Schimpf 2017; Boston and Cimpian 2018; García-Peñalvo et al. 2016; Nash 2017). At the upper-secondary level, many of the interventions are designed to increase computing-related knowledge and confidence (Main and Schimpf 2017; Gürer and Camp 2002; Siiman et al. 2014; Milam 2012; Menon et al. 2019; Brotman and Moore 2008; Murphy et al. 2019; Crick 2017). Both, however, aim at increasing engagement and interest in computing as the key theme.

The research by Gürer and Camp (2002) shows that teachers and classrooms that do not make explicit efforts to provide a female-friendly environment for exploring computing will naturally end-up promoting computing as a male-oriented domain. It is being acknowledged as a result of the differences in leisure-time preferences among girls vs. boys (Main and Schimpf 2017). In effect of that, boys, who are on average one year ahead of girls in computer usage, according to the findings tend to monopolize the instructor's time, computer labs, and the curriculum material (Siiman et al. 2014; Willoughby 2008; Brotman and Moore 2008). Girls, often feeling less competent, take the position of a guest in the computer lab, and many tend to prefer to sit back and let boys take over. This situation leads to even fewer opportunities to gain experience and increase their confidence with computers, which can be observed among girls as well as among less experienced boys. When students, whether girls or boys, fall into this vicious circle (Wieselmann et al. 2020), it is not very likely they escape it without the explicit initiative of the school and parents who would create an engaging environment effectively pulling these kids back in CS education. As girls fall in this vicious circle more often than boys, it is natural to ask, what the girl-friendly CS education environment looks like, what requirements it poses on the curriculum, culture, and the overall environment (RQ3). Teachers need to implement preventive measures to ensure that no group of students can monopolize lessons based solemnly on their preferences (Barker and Aspray 2006). Works exist that examine the benefits of students' separation into groups within the classroom, allowing educators to tailor CS education to meet students' needs best.

Research suggests that the separation of classes based on gender (Rec.4.1.) (Gürer and Camp 2002; García-Peñalvo et al. 2016; Barker and Aspray 2006) has a similar effect as separation based on previous experience (Rec.4.2.) (Siiman et al. 2014). This needs further investigation. All-girls classrooms are shown to be beneficial for adolescent girls (as well as adult women) (Buhnova et al. 2019a, b) to create a safe environment when entering CS education and building the initial confidence, making it easier to experiment and expressing their creativity freely. 
Moreover, many girls might find it easier to find their way to technology in homogeneous girl groups, while in mixed groups their self-image might be a hindrance (García-Peñalvo et al. 2016). On the other hand, this measure might not be easy to implement. Schools are likely to face challenges connected to the practicality of gender segregation in terms of organizing girls-only classes. The practicality may be further challenged by the fact that in elective CS education, very few girls tend to elect the subject. To overcome the practicality burden, segregation-by-experience could serve the purpose in classes where segregation-by-gender is not practically feasible. Existing research suggests that the learning environment and the signals girls receive in the classroom play a critical role in determining their interest in computing (Boston and Cimpian 2018). The strategies to make the environment less hostile for girls are of enormous impact. According to the examined literature, educators need to work to diminish the usual informal hierarchy and defensive climate based on computing skills, which may take place in CS education (Main and Schimpf 2017), and need to install a growth mind-set (Rec.5.2.) (Boston and Cimpian 2018), where everyone can genuinely believe that they can improve, having a positive and constructive attitude toward failure (Rec.5.3.) (Boston and Cimpian 2018), with the failure being seen as an opportunity to improve.

Girls' leisure-time preferences show not only that girls are statistically better in collaborative and social tasks, but they prefer to participate in such activities more (Nash 2017). This opens a way to provide low-stakes opportunities (Rec.5.1.) (Boston and Cimpian 2018; Nash 2017) for girls to succeed in solving the classroom tasks and see themselves as contributors to the solution. This could be achieved e.g., by creating more opportunities for discussion and presentation of solution design, making these skills (which are also seen as crucial for success in computing career) an integral part of the feeling of success in computing (Boston and Cimpian 2018) . It seams that if the competences in which girls tend to excel become part of the CS education design, we can stimulate and facilitate the identification of girls with computing even for girls who might be stronger in other skills than which we stereotypically link to computing. This is actually in alignment with the situation in computing industry, where a diverse cohort of individuals is needed to build various teams, involved in product design, implementation, testing, management, making the soft skills of similar importance than the hard technical skills for success in computing (Rec. 3.4.) (García-Peñalvo et al. 2016).

The fact that differences in leisure-time activities at a very young age influence stance towards computing later in life results in efforts to provide each child with appealing purposeful early contact with a computer or computing-promoting toys (Rec.3.1.) (Main and Schimpf 2017; Milam 2012). Girls usually start using a computer much later, for homework, research and socializing; while at that time, boys already tend to have a few more years of experience with computers (Main and Schimpf 2017), which makes it hard to reverse differences in computing literacy. This may be attributed to the fact that what makes an appealing first contact with computers for boys is not that appealing for girls; girls hence opt-out from this first attraction and start exploring computing later (Main and Schimpf 2017).

Little research exists on what could be an appealing first contact with computers for girls. A successful example, which is, however, connected to a slightly later age, 
are interdisciplinary explanatory activities, such as animations, graphics, and photography (Siiman et al. 2014; García-Peñalvo et al. 2016). Girls also tend to be good at typical school achievements, e.g., using a computer for homework and writing assignments (Siiman et al. 2014; Main and Schimpf 2017). Hence they tend to be attracted to computing once they understand it as a tool impacting their potential academic achievements in all disciplines as a way to test hypotheses and a resource of new knowledge (Siiman et al. 2014). Attempts should be made to help girls understand these benefits of computing early, showing them computing as a facilitator towards their goals and activities that are naturally appealing to them (Main and Schimpf 2017; Siiman et al. 2014; Boston and Cimpian 2018; Milam 2012). With this, the educators can even start offline (Rec.3.2.) (García-Peñalvo et al. 2016; Menon et al. 2019), or well designed appealing assignments in visual programming environments (Rec.3.3.) (Siiman et al. 2014; Menon et al. 2019).

In the primary education phase, the time spent using a computer is similar for both genders, sometimes even with longer periods of time spent by girls (Main and Schimpf 2017). However, this changes in 5th or 6th grade, at the upper-secondary level, when the statistics of attitudes, perception, and usage of computers among girls, and some boys as well, radically change and increasingly state that they do not intend to pursue a degree in CS education as they prefer other subjects (Main and Schimpf 2017). The mentioned interventions on upper-secondary (high-school) level include providing girls with exciting and engaging hands-on experiences, increasing motivation to pursue computing through emphasizing the interdisciplinary nature and the social impact of computing work (Main and Schimpf 2017; Siiman et al. 2014; García-Peñalvo et al. 2016; Brotman and Moore 2008; Murphy et al. 2019; Crick 2017; Nash 2017), introducing girls to positive role models (Boston and Cimpian 2018) (Rec.1.1.-4.) and providing information to teachers to encourage interest in computing (Rec.6.1.-3.).

Wider adoption of these pedagogical strategies seems to have the potential to significantly increase the interest, participation, and retention of girls in computing (Main and Schimpf 2017; Nash 2017; Siiman et al. 2014; García-Peñalvo et al. 2016; Brotman and Moore 2008; Murphy et al. 2019; Crick 2017).

Siiman et al. (2014) analyzed successful extracurricular activities and concluded that the provision of pedagogically effective extracurricular activities in the long term requires unsustainably high effort. It usually either results in ending the activity too early, or in limiting the effort to a one-shot intervention with no long-term effect. The study pointed out that the integration of such activities in a regular CS education classroom is a goal without which a change can hardly be achieved. However, to reach this goal, professional teacher development programs need to be introduced that equip the teachers with knowledge, easy-to-use tools, and guidelines (Rec.6.1.). The teachers need this guidance to build up the necessary confidence, and hence, be able to transfer their confidence to motivate students into a career in computer science (Brotman and Moore 2008; Nash 2017; Crick 2017; Siiman et al. 2014).

The most common approach to teaching computer science is to gradually engage in programming through a process of solving tasks, from very simple to more complex ones (García-Peñalvo et al. 2016). For many girls, this process might make it very difficult to achieve real intellectual satisfaction, which may be a significant 
obstacle in retaining girls in computing, as this way of thinking degrades digital literacy to pure coding literacy (García-Peñalvo et al. 2016). Computer science is, however, not only about coding. It requires fundamental skills, such as creativity, imagination, innovation, solution design and problem-solving, understanding of human behavior and needs, experience design, and a combination of mathematical and engineering thinking to use concepts of computer science effectively (Main and Schimpf 2017; García-Peñalvo et al. 2016). An endeavor to present computer science as a tool to realize and scale any idea, originating from and innovating any discipline possible and available to anyone independent of previous coding expertise, seams to be the key to bring girls to understand the opportunities in computing and space for their creativity. There is no silver bullet to solve this problem, and it will not merely fix itself with time. A well-designed package of interventions is needed in each classroom for all the students who otherwise miss on the opportunities provided by computing in any discipline.

In response to RQ3, we looked for consistently recommended measures among the reviewed literature. Our literature review shows that courses considered as effective in the reviews used similar strategies. We extracted and summarised these strategies and measures that emerged as especially promising in Table 2 . Thus, courses seem to be effective when they include at least the following aspects, with an explicit effort to include them as early as possible within a long-term CS education classroom and curriculum design:

1. use inquiry-based and real-world learning activities to engage students in computing (in 7 from 11 reviews) (Brotman and Moore 2008; Main and Schimpf 2017; Nash 2017; Siiman et al. 2014; Crick 2017; García-Peñalvo et al. 2016; Murphy et al. 2019),

2. show as many facets and interdisciplinary applications of computer science as possible, as early as possible, to attract students from diverse disciplines (in 6 from 11 reviews) (Brotman and Moore 2008; Crick 2017; García-Peñalvo et al. 2016; Main and Schimpf 2017; Murphy et al. 2019; Siiman et al. 2014),

3. split classes at best by experience, or at least by gender or by being part of the same interest group (in 5 from 11 reviews) (García-Peñalvo et al. 2016; Gürer and Camp 2002; Boston and Cimpian 2018; Brotman and Moore 2008; Nash 2017),

4. give more emphasis on the process of thinking, designing and problem-solving than the actual coding (in 4 from 11 reviews) (García-Peñalvo et al. 2016; Menon et al. 2019; Nash 2017; Boston and Cimpian 2018),

5. use visual programming environments to teach introductory programming (in 4 from 11 reviews) (Main and Schimpf 2017; Menon et al. 2019; Milam 2012; Siiman et al. 2014),

6. take students to events and excursions, share with them stories and role models from the history of computing (in 4 from 11 reviews) (Boston and Cimpian 2018; Brotman and Moore 2008; Nash 2017; Gürer and Camp 2002).

As these recommendations were directly or indirectly mentioned in the majority of publications, it provides a set of recommendations that appear to impact the retention 
of girls in CS education most and would be minimally required when implementing a successful CS course.

\section{Discussion}

Our aggregated umbrella review revealed some gaps in existing research on the topic (RQ4), which are connected to the segregation of causes and solutions in existing studies, as well as missing easy-to-use tools and guidelines on gender-sensitive CS education, i.e., consciously working with the awareness that each education style might have different effectiveness for each gender (Diller 2018). This section provides discussion of our observations, together with outlining directions for further studies to advance the state of the art.

\subsection{Segregation of causes and solutions}

An important finding from our aggregated umbrella review is that the peer-reviewed research is focusing mostly on finding the cause of a gender discrepancy, while the search for the solution is primarily lead by the industry (see Table 1). This is manifested by the growing number of programs offered to girls by industry. The research papers state hypotheses on causes and their consequences. The papers introducing solutions, however, often do not care so much about the cause; they observe the consequences of it and related problems and react by introducing solutions and strategies assumed to help. However, it is unclear if they understand and have in mind the possible cause of the problem and thus, if the cure helps the patient.

The shortcomings of existing research on the topic of interventions to recruit and retain girls allow only for tentative and potential solutions, which were not systematically tested. The research articles describing interventions usually do not conduct rigorous statistical comparisons between treatment and control groups (Siiman et al. 2014). Moreover, there are very few peer-reviewed intervention studies on the topic, as reported by Lye and Koh (2014), and confirmed with our umbrella review. Also, these few existing studies have mostly been conducted in extracurricular activities, which makes them a limited example when it comes to the representativeness of a typical CS classroom.

\subsection{Challenges of gender-sensitive CS education}

One of the major themes in the existing reviews is the fact of missing guidelines on gender-sensitive CS education. This includes not only missing tools and guidance on the curriculum design, but also lacking teachers awareness of insights of neurological science and tendencies that may be naturally more visible in each gender. There also seems to be a lack of guidance on how to benefit from students' diversity in the CS classroom and prevent masculine heteronomy while some male teachers might provide learning environment (without being aware of it) that benefit male students more (Annis and Nesbitt 2017; Main and Schimpf 2017; García-Peñalvo et al. 2016; 
Boston and Cimpian 2018; Nash 2017). This might make many girls feel less competent than boys, although their chances to succeed in computing careers might be the same (see the discussion in Section 3 on the competencies needed for success in the industry, and their difference to what we teach and grade in classrooms).

The differences in gender tendencies, described in psychology by the Bell curve of gender tendencies (Annis and Nesbitt 2017), also explain why diverse teams are more successful than homogeneous ones. For instance, in case of problem-solving, girls have a higher tendency to define the problem in broader terms and allow a wider array of potential factors (Brizendine L 2006; Zaidi 2010), while boys have higher tendencies to approach the problem by eliminating and isolating issues, and approaching them in isolation (Fisher H 1999). When girls create their map of the problem space, which tends to holistically characterize the domain to a greater detail as a web of factors (allowing in also past experiences, as they have neurologically higher memory capacity for past events than boys) (Blum 1998), they might appear to be stuck and feel anxiety when being pushed by the teacher to move on quickly (Decety and Jackson 2004). None of these two problem-solving strategies is better or worse. They work best in combination (Annis and Nesbitt 2017). However, when CS education is strongly male-dominated, the teachers are likely to fall in the stereotype linking successful solutions with the problem-solving strategy more common for boys.

The mentioned, predominantly female tendencies for holistic thinking and abilities to organize many pieces of information in complex patterns, together with seeing more relationships between these patterns, make them more attracted to multidisciplinary applications of computing. However, these complex multidisciplinary applications are nowadays getting very little space in CS education. Another important determinant in building gender-sensitive CS education is the understanding of the importance of testosterone (dominant male hormone) in boys' drive and competitiveness (Kivlighan et al. 2005). For many girls, learning-by-competition is naturally uncomfortable, hindering their ability to achieve their best results.

In addition, the fact that many girls prefer a safer environment without pressure, competition, and with more time for their computing assignments (Buhnova et al. 2019a, b), might be misinterpreted by the teacher as the fact that these girls are weaker in computing, which is not necessarily the case (Buhnova and Happe 2020). When girls are given the conditions they need to master computing, they might achieve exceptional results, and later excel in the industry. Hence computing teachers must have this knowledge and make a deliberate effort to reflect it in their curriculum, e.g., by pre-designing it for multi-speed learning.

Overall, it should be considered that neither the group of girls nor the group of boys is homogeneous in itself. The results and interpretations refer to conceptually typical girls or boys, well aware that all children are different and that gender attributions may be manifested through social constructions.

\subsection{Bridging of domains and fusion of insights}

When searching for inspiration in addressing the discussed challenges, there seems to be enormous potential in the overlap between initiatives redesigning curriculum and potential initiatives rethinking inclusive and gender-sensitive CS education. 
The research examining changes of curricula and other reforms would benefit from imagining measures in the curriculum to target previously non-integrated skill sets, perception of identity, and belonging. On the other hand, the researchers targeting inclusive pedagogy, gender balance, and stereotypes would benefit from outlining what it means when translated into actual teaching practices and integrated into the curriculum. To this end, some of the future research questions that could bring valuable insights include:

- What insights can we gain from exploring more deeply the experiences of girls who do enjoy and engage in CS education at various ages?

- What insights can we gain from girls who disengaged from CS education during their education or after?

- What insights can we gain from exploring the experiences of girls who do enjoy and engage in other domains of science, technology, engineering, and mathematics (STEM)?

- What insights can we gain from understanding identity and belonging formation for girls across various fields of science, technology, engineering, and mathematics (STEM)?

\section{Conclusions}

Our aggregated umbrella literature review points to some exciting challenges in the field of CS education. Computer science encompasses many perspectives: it is creative, social, and provides for well-paid and often interdisciplinary careers. Future CS education research needs to demonstrate the usefulness of CS in contributing to solving critical societal, natural, and economic challenges. There are many interesting opportunities for women to engage in this field.

Several programs, initiatives, and interventions have been launched to address women's underrepresentation in computer science. At the high-school level, several studies observe differences in computer science course behavior between male and female adolescents (Main and Schimpf 2017; García-Peñalvo et al. 2016; Boston and Cimpian 2018; Nash 2017). Girls are observed to be less likely to choose computer science courses due to classroom environments perceived by them as hostile to their way of self-expression and achievement understanding. The related stereotypical images and messages, as well as individual interactions in the classroom, reduce girls' sense of belonging. The effective intervention strategies often include providing girls with engaging hands-on experiences, increasing motivation to pursue computing through emphasizing the social impact of computing work, providing for girls opportunities to succeed with their strengths, introducing girls to positive but relatable role models, and providing training to teachers and key individuals to encourage greater interest in computing and technology. If given the necessary support through professional development programs, educators could react and adapt. For the moment, CS education might be the essential factor that can be changed to influence the recruitment and retention of girls in computing. Yet, sufficient pedagogical guidance is essential to make this endeavor successful. 
Our umbrella review of reviews shows that although numerous reviews on this topic exist, there is no comprehensive and current evaluation of state of the art in interventions fostering girls' interest in CS education that would span across all relevant factors, which we have extracted from the individual reviews.

Current reviews do not thoroughly analyze and explain the causes and consequences of gender distribution in CS, and they as well do not identify effective interventions formulated as pedagogical guidance (Craig 2016; Trauth 2017; Loiacono et al. 2016).

Acknowledgements The writing of this article was supported in part by Vector Stiftung, Project "Mädchen für Informatik begeistern" at Karlsruhe Institute of Technology (KIT).

Funding Open Access funding enabled and organized by Projekt DEAL.

\section{Compliance with Ethical Standards}

Conflict of interests The authors declare that no conflicts of interest or competing interests exist.

Open Access This article is licensed under a Creative Commons Attribution 4.0 International License, which permits use, sharing, adaptation, distribution and reproduction in any medium or format, as long as you give appropriate credit to the original author(s) and the source, provide a link to the Creative Commons licence, and indicate if changes were made. The images or other third party material in this article are included in the article's Creative Commons licence, unless indicated otherwise in a credit line to the material. If material is not included in the article's Creative Commons licence and your intended use is not permitted by statutory regulation or exceeds the permitted use, you will need to obtain permission directly from the copyright holder. To view a copy of this licence, visit http://creativecommonshorg/licenses/by/4. $0 /$.

\section{References}

Accenture (2016). Cracking the gender code: Get 3x more women in computing. Available online at https://www.accenture.com/us-en/cracking-the-gender-code.

Ainley, M. (2007). Being and feeling interested: Transient state, mood, and disposition. In Emotion in education (pp. 147-163): Elsevier.

Annis, B., \& Nesbitt, R. (2017). Results at the Top: Using Gender Intelligence to Create Breakthrough Growth: Wiley.

Aromataris, E., Fernandez, R., Godfrey, C. M., Holly, C., Khalil, H., Tungpunkom, P. (2015). Summarizing systematic reviews: methodological development, conduct and reporting of an umbrella review approach. International Journal of Evidence-Based Healthcare, 13(3), 132-140.

Barker, L.J., \& Aspray, W. (2006). The state of research on girls and IT. In Women and Information Technology: Research on Underrepresentation: The MIT Press.

Blum, D. (1998). Sex on the Brain: The Biological Differences Between Men and Women: Penguin Books.

Boston, J. S., \& Cimpian, A. (2018). How do we encourage gifted girls to pursue and succeed in science and engineering? Gifted Child Today, 41(4), 196-207.

Brizendine L (2006). The Female Brain: Morgan Road Books.

Brotman, J. S., \& Moore, F. M. (2008). Girls and science: a review of four themes in the science education literature. Journal of Research in Science Teaching: The Official Journal of the National Association for Research in Science Teaching, 45(9), 971-1002.

Buhnova, B., \& Prikrylova, D. (2019a). Women want to learn tech: Lessons from the czechitas education project. In 2019 IEEE/ACM 2Nd international workshop on gender equality in software engineering (pp. 25-28). GE: IEEE. 
Buhnova, B., Jurystova, L., Prikrylova, D. (2019b). Assisting women in career change towards software engineering: experience from czechitas ngo. In Proceedings of the 13th European Conference on Software Architecture-Volume (vol 2, pp. 88-93).

Buhnova, B., \& Happe, L. (2020). Girl-friendly computer science classroom: Czechitas experience report. In European Conference on Software Architecture (pp. 125-137): Springer.

Bull, A., Gilbert, J., Barwick, H., Hipkins, R., Baker, R. (2010). Inspired by science. A paper commissioned by the Royal Society of New Zealand and the Prime Minister's Chief Science Advisor Accessed on the, 20(03), 2013.

Craig, A. (2016). Theorising about gender and computing interventions through an evaluation framework. Information Systems Journal, 26(6), 585-611. https://doi.org/10.1111/isj.12072.

Crick, T. (2017). Final draft: Computing education: An overview of research in the field.

Decety, J., \& Jackson, P. L. (2004). The functional architecture of human empathy. Behavioral and Cognitive Neuroscience Reviews, 3(2), 71-100.

Diller, A. (2018). The gender question in education: Theory, pedagogy, and politics: Routledge.

Fisher H (1999). The first sex: The natural talents of women and how they are changing the world: Ballantine Books.

García-Peñalvo, F., Reimann D, Tuul M, Rees A, Jormanainen I (2016). Taccle 3, o5: An overview of the most relevant literature on coding and computational thinking with emphasis on the relevant issues for teachers: Belgium. https://doi.org/10.5281/zenodo 165123.

Gorbacheva, E., Beekhuyzen, J., vom Brocke, J., Becker, J. (2019). Directions for research on gender imbalance in the it profession. European Journal of Information Systems, 28(1), 43-67.

Gough, D.A., Oliver, S., Thomas, J. (2013). Learning from research: systematic reviews for informing policy decisions: a quick guide: Nesta London.

Gürer, D., \& Camp, T. (2002). An acm-w literature review on women in computing. SIGCSE Bull, 34(2), 121-127. https://doi.org/10.1145/543812.543844.

Kivlighan, K. T., Granger, D. A., Booth, A. (2005). Gender differences in testosterone and cortisol response to competition. Psychoneuroendocrinology, 30(1), 58-71.

Krapp, A., \& Prenzel, M. (2011). Research on interest in science: theories, methods, and findings. International Journal of Science Education, 33(1), 27-50.

Loiacono, E. T., Iyer, L. S., Armstrong, D. J., Beekhuyzen, J. P., Craig, A. (2016). Ais women's network: Advancing women in is academia. CAIS, 38, 38 .

Lye, S. Y., \& Koh, J. H. L. (2014). Review on teaching and learning of computational thinking through programming: What is next for k-12? Computers in Human Behavior, 41, 51-61.

Main, J. B., \& Schimpf, C. (2017). The underrepresentation of women in computing fields: a synthesis of literature using a life course perspective. IEEE Transactions on Education, 60(4), 296304.

Menon, D., Bp, S., Romero, M., Viéville, T. (2019). Going beyond digital literacy to develop computational thinking in $k$-12 education: Smart Pedagogy In Digital Learning.

Milam, J. (2012). Girls and stem education: a literature review. Atlanta: Georgia Institute of Technology.

Murphy, A., Kelly, B., Bergmann, K., Khaletskyy, K., O’Connor, R.V., Clarke, P.M. (2019). Examining unequal gender distribution in software engineering. In European Conference on Software Process Improvement (pp. 659-671): Springer.

Nash, J. (2017). Understanding how to interest girls in stem education: a look at how lego® education ambassador teachers engage female students in stem learning. $\mathrm{PhD}$ thesis, University of Florida.

Powell, C., \& Chang, A.M. (2016). Women in tech as a driver for growth in emerging economies: Council on Foreign Relations.

Schlegel, F. (2016). Unesco science report towards 2030 Available online at https://unesdoc.unesco.org/ ark:/48223/pf0000235406.

Siiman, L. A., Pedaste, M., Tõnisson, E., Sell, R., Jaakkola, T., Alimisis, D. (2014). A review of interventions to recruit and retain ict students. International Journal of Modern Education and Computer Science, 6(3), 45.

Trauth, E. (2017). A research agenda for social inclusion in information systems. ACM SIGMIS Database: the Database for Advances in Information Systems, 48(2), 9-20.

Wieselmann, J.R., Dare, E.A., Ring-Whalen, E.A., Roehrig, G.H. (2020). "i just do what the boys tell me": Exploring small group student interactions in an integrated stem unit. Journal of Research in Science Teaching, 57(1), 112-144. 
Willoughby, T. (2008). A short-term longitudinal study of internet and computer game use by adolescent boys and girls: prevalence, frequency of use, and psychosocial predictors. Developmental Psychology, 44(1), 195.

Zaidi, Z. F. (2010). Gender differences in human brain: a review. The Open Anatomy Journal, 2(1).

Publisher's note Springer Nature remains neutral with regard to jurisdictional claims in published maps and institutional affiliations. 